\title{
The Effect of the Pre-Conditioning Step on the Shear Bond Strength of Nano-Filled Resin-Modified Glass-lonomer to Dentin
}

Farid S El-Askary

Mohammed S Nassif ${ }^{b}$

\section{ABSTRACT}

Objectives: The purpose of this study was to evaluate the effect of the pre-conditioning step using different dentin conditioners on the shear bond strength (SBS) of the nano-filled resin-modified glass ionomer (RMGI) to dentin.

Methods: Twenty-four human molars were used in this study. Subsequent to teeth sectioning in the mesio-distal direction and creation of the smear layer, the teeth were divided into six groups with and without the pre-conditioning step. Dentin surfaces were restored with RMGI cylinders and the specimens were stored in distilled water at $37^{\circ} \mathrm{C}$ for 24 hours. SBS test were performed using a Universal Testing Machine at a crosshead speed of $0.5 \mathrm{~mm} /$ minute. Statistical analysis was performed using One-Way ANOVA followed by Duncan's Multiple Range Test. RMGI/dentin interface was evaluated using SEM operated at $12 \mathrm{Kv}$ at 2000X magnification.

Results: $35 \%$ phosphoric acid and EDTA yielded significantly higher SBS $112.0 \pm 1.8$ and $11.9 \pm 4.4$ $\mathrm{MPa}$, respectively), compared to all tested groups. In addition, $25 \%$ polyacrylic acid (8.6 $\pm 3.1 \mathrm{MPa}$ ) reported significantly higher SBS than the nano-filled RMGI when applied according to manufacturer instructions (5.5 $\pm 2.2 \mathrm{MPa}$ ). When nano-filled RMGI was applied without its nano-primer, or directly over $25 \%$ polyacrylic acid conditioned dentin exhibited $100 \%$ pre-test failure, and their SBS were expressed as $0.0 \pm 0.0 \mathrm{MPa}$. SEM revealed that the nano-primer was unable to decalcify and hybridize the dentin surface.

Conclusions: Based on the results of the current study, the pre-conditioning step effectively improves the SBS of nano-filled RMGI to dentin. The self-adhesiveness of nano-filled RMGI was not proved in this study. (Eur J Dent 2011;5:150-156)

Key words: Dentin conditioners; Bond strength; Nano-filled resin-modified glass-ionomer.

- a Department of Operative Dentistry, Faculty of Dentistry, Ain Shams University, Cairo, Egypt.

b Biomaterial Department, Faculty of Dentistry, Ain Shams University, Cairo, Egypt.

- Corresponding author: Farid S El-Askary Department of Operative Dentistry, Faculty of Dentistry, Ain Shams University, Cairo, Egypt.

Phone: +20224196956

Fax: +20226820191

E-mail: faridelaskarylahotmail.com.

\section{INTRODUCTION}

Since Wilson and Kent's introduction of glass ionomer cement (GIC) during the early 70's, several modifications have been made to improve its properties. ' Glass ionomer cements are considered the most effective biomaterial that bond to hard tooth substrates. ${ }^{2,3}$ Comparing the retention rates of class $\mathrm{V}$ adhesive restorations, glass iono- 
mers showed the most effective and durable bond to tooth structure. ${ }^{4}$

Resin-modified glass ionomer (RMGI) is a combination of glass ionomer fillers and watersoluble monomer in an aqueous solution of polyacrylic acid. ${ }^{5}$ While conventional Gls bond to tooth substrate by ion-exchange, ${ }^{6}$ RMGIs bond to tooth substrates through both ion-exchange and micromechanical interlock. ${ }^{7,8}$

A newly introduced nano-filled RMGI restorative (Ketac Nano-100, 3M-ESPE) undergoes both glass ionomer and free radical reactions. According to its manufacturer, “Ketac Nano-100 restorative is a new technical development that combines the benefits of a resin modified light cure glass ionomer and bonded nano-filler technology". Nano-filled RMGI is a light-cured paste/ paste system that is delivered from a multi-dose "ClickerTM Dispenser". Its chemistry is based on metacrylate modified polyalkenoic acid, which was firstly employed in Vitrebond liner/base material (Ketac N-100, Technical product profile, 3 M-ESPE).

Nano-primer is a one-component acidic primer, which is essential to bond the nano-filled RMGI to dentin (Ketac Nano-100 technical product profile) and it does not require the pre-conditioning step of the dentin surface. ${ }^{3}$ The manufacturer claims that the bond strength of nano-filled RMGI to dentin using the nano-primer is equivalent to the greatest number of Gls used. This claim was not confirmed in the study by Coutinho et al. ${ }^{3}$ Conventional RMGI (Fuji II LC) showed a significantly higher $\mu$ TBS to both enamel and dentin than that recorded with nano-filled RMGI.

The interaction of acids to tooth structure has been evaluated extensively; nevertheless, it has never been elucidated. ${ }^{9}$ The use of different dentin conditioners did not only improve the bond strength of self-etching primers to dentin, ${ }^{10,11}$ but also improved the shear bond strength (SBS) of the glass ionomer adhesive. ${ }^{12}$ Accordingly, the purpose of this study was to evaluate the effect of the pre-conditioning step on the SBS of nanofilled RMGI to dentin. The null hypothesis tested is that the pre-conditioning step will not affect the SBS of nano-filled RMGI to dentin.

\section{MATERIALS AND METHODS}

Twenty-four caries-free non-restored hu- man molars were used in this study. The teeth were collected and stored in a $0.1 \%$ thymol solution for not more than one month. Each tooth was sectioned in a mesio-distal direction down to the root into two halves, using a low-speed diamond disc under copious water irrigation; then, the two halves were completely separated using a surgical chisel, ${ }^{12}$ yielding 48 halves. Each half was fixed from its dentin side to the base of a metallic rectangular mould with double-faced adhesive tape. Auto-cured acrylic resin material (Acroston, under exclusive license of Acroston Dental Company-Cairo, Egypt) was poured to completely cover each half. After hardening of the acrylic resin, each dentin surface was wet ground using 600 grit $\mathrm{SiC}$ papers for 60 seconds to create a standardized smear layer.

\section{Experimental procedures}

The 48-halves were equally divided into 6 groups ( $n=8$ ) according to the dentin surface treatment procedures. Materials, compositions and manufacturers are summarized in Table 1. Before the application of each dentin treatment, doublefaced adhesive tape, with a hole of $3 \mathrm{~mm}$ in diameter was fixed over the dentin surface to limit the bonded surface area.

Group 1: The nano-filled RMGI was applied according to manufacturer instructions. Nanoprimer was applied for 15 seconds, air-dried using gentle, compressed-air for 10 seconds, and light cured for 10 seconds with a halogen light-curing unit (Hilux, Dental Curing Light Unit, Benlioglu; Ankara, Turkeyl with an output of $600 \mathrm{~mW} / \mathrm{cm}^{2}$. Before the application of the nano-filled RMGI, a split Teflon mould, $2 \mathrm{~mm}$ in thickness with a central hole of $3 \mathrm{~mm}$ in diameter, was fixed over the dentin surface. The material was dispensed over a pad with the aid of its Clicker ${ }^{\top M}$ Dispenser, mixed using a metallic spatula for 20 seconds, and applied over the cured primer, then, the material was light cured for 20 seconds.

Group 2: Nano-filled RMGI was directly applied over the dentin surface, without the application of the nano-primer and light cured as in Group 1.

Group 3: The dentin surface was conditioned using $25 \%$ polyacrylic acid for 10 seconds, rinsed with a copious air/water spray for 10 seconds and, blot dried using sterile cotton, leaving the dentin surface visibly moist (wet bonding). Nano-filled 
RMGI was applied directly over the dentin surface, without the application of the nano-primer and light cured, as in Group 1.

Group 4: The dentin surface was conditioned with $25 \%$ polyacrylic acid as in Group 3. Nanoprimer and nano-filled RMGI were applied as described in Group 1.

Group 5: The dentin surface was etched using $35 \%$ phosphoric acid for 15 seconds, rinsed for 20 seconds and blot dried as in Group 3. Nano-primer and nano-filled RMGI were applied as described for Group 1.

Group 6: The dentin surface was conditioned with EDTA solution for 60 seconds, rinsed for 20 seconds and blot dried as in Group 3. Nano-primer and nano-filled RMGI were applied as described for Group 1.

Subsequent to light curing of the nano-filled RMGI, the Teflon mould was dissembled, and the dentin surfaces- with their attached RMGI cylinders- were stored in distilled water for 24 hours at $37^{\circ} \mathrm{C}$.

\section{SBS testing}

After 24 hours, each specimen was mounted to the universal testing machine (LR5K series, Lloyd Instrument; Fareham, UK). A specially fabricated, metallic, chisel-bladed instrument was positioned as accurately as possible on the nano-filled RMGI/ dentin interface. The test was run at a crosshead speed of $0.5 \mathrm{~mm} / \mathrm{minute}$ until failure. The load recorded in Newton was divided over the calculated surface area and the SBS was retrieved in MPa. Groups 2 and 3 were not tested, as all specimens of these two groups failed before testing during the 24-hour storage period, and their SBS was expressed as 0.0 MPa for statistical analysis.

\section{SEM evaluation}

An additional eight human molars were included to evaluate the nano-filled RMGI/dentin interface for Groups 1, 4, 5 and 6, two teeth per group. The occlusal enamel of each tooth was wet ground using the laboratory trimmer to expose a flat dentin surface surrounded by enamel. Subsequent to enamel removal, the dentin surfaces were wet ground using 600 grit SiC paper for 60 seconds, in order to create a standardized smear layer. The teeth of the above mentioned groups (Groups 1, 4, 5 and 6) were treated as described for SBS testing. After each treatment, an approximately 2 $\mathrm{mm}$ thick nano-filled RMGI block was applied and light cured for 20 seconds. After 24-hour storage period in distilled water at $37^{\circ} \mathrm{C}$, each tooth was sectioned mesio-distally using the low speed diamond disc under copious water irrigation to the level of the cervical line into two halves. Then, the two halves were separated by an additional horizontal cut. Two specimens were obtained from each tooth (four specimens per group). The evaluated surfaces were wet ground with 600, 800, 1000 grits and finally with 1200 grit SiC papers.

The evaluated surfaces were acid etched with $35 \%$ phosphoric acid for 15 seconds, rinsed for 20 seconds and, then, gently air dried. The specimens were immersed in 5\% sodium hypochlorite for 120 seconds, and washed under running water for 5 minutes. The specimens were dehydrated in ascending concentration of ethanol, 50\%, 70\%, $90 \%$ for 20 minutes each and, then immersed in $100 \%$ for 1 hour. The specimens were left to dry on absorbent paper in closed container over night.

The specimens were fixed on specimen's holder using double-faced stickers and sputter coated (BAL-TEC, SCD 005 sputter coater, Germany). Nano-filled RMGI/dentin interface was evaluated at 2000X using SEM (Philips XL series, XL30, Holland) operated at $12 \mathrm{Kv}$.

All data of SBS in MPa was expressed as mean \pm standard deviation (SD). Statistical analysis was carried out using SAS program (SAS, 1988). One Way Analysis of Variance (Procedure ANOVA of SAS) followed by Duncan's Multiple Range Test were used to test the effect of dentin conditioners on SBS.

\section{RESULTS}

SBS results for the effect of different dentin conditioners are shown in Table 2. One way ANOVA followed by Duncan's Multiple Range Test revealed that the pre-conditioning step using $35 \%$ phosphoric acid and EDTA solution (Groups 5 and 6 , respectivelyl yielded statistically significant higher SBS compared to all tested groups

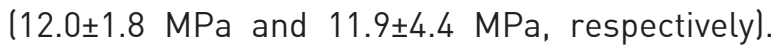
No statistical significant was recorded between these two above groups. When the dentin was preconditioned with $25 \%$ polyacrylic acid (Group 4, 8.6 $\pm 3.1 \mathrm{MPa}$ ), it reported a statistically significant higher SBS than that when the nano-filled RMGI 
was applied according to manufacturer instructions (Group 1, 5.5 $\pm 2.2 \mathrm{MPa}$ ). SBS of nano-filled RMGI when directly applied either over dentin surface (Group 2) or over conditioned dentin with $25 \%$ polyacrylic acid (Group 3) showed significant differences between all groups $10.0 \pm 0.0 \mathrm{MPa}$ and $0.0 \pm 0.0 \mathrm{MPa}$, respectively), which exhibited $100 \%$ pre-test failure in all their specimens. No significant differences were observed between the two above groups.

\section{SEM evaluation}

SEM evaluation of nano-filled RMGI/dentin interface, when the material was applied according to manufacturer instructions (Group 1) revealed no evidence of hybrid layer formation or resin tag extensions. A gap was detected between the restoration and underlying dentin, and the smear layer was observed over the dentin surface (Figure 1). When the dentin surface was pre-conditioned with $25 \%$ polyacrylic acid prior to the use of the nano-primer (Group 4), few discrete and very shallow resin tags extensions were noticed, although a thin hybrid layer was observed (Figure 2).
Numerous long, funnel-shaped, resin tag extensions were detected when the dentin surface was pre-conditioned with $35 \%$ phosphoric acid prior to the application of the nano-primer (Group 5). A thick hybrid layer was evident with filler distribution at the bottom and within the hybrid layer, as well as around the orifices of the dentinal tubules (Figure 3). Long and thin resin tag extensions were noticed when the dentin surfaces were pre-conditioned with EDTA solution for 60 seconds prior to the application of the nano-primer (Group 6). The hybrid layer was thinner than that detected with Group 5, with a few discrete filler distributions (Figure 4).

\section{DISCUSSION}

Self-adhesiveness properties to tooth structure $^{3}$, anticariogenicity ${ }^{13}$ and minimal tooth/restoration interface microleakage ${ }^{14}$ are among the advantages presented by glass ionomer cements. The introduction of RMGIs was aimed to overcome the drawbacks encountered with conventional Gls. ${ }^{15}$ A new approach to improve some properties of conventional RMGls is the introduction of

Table 1. Materials used in this study, their compositions and their manufacturers.

\begin{tabular}{|c|c|c|}
\hline Materials & Compositions & Manufacturers \\
\hline $\begin{array}{l}\text { Ketac Nano-100 } \\
\text { (nano-filled RMGI) }\end{array}$ & $\begin{array}{c}\text { Silane treated glass } 40-50 \% \text {, silane treated } \\
\text { zirconia oxide silica } 20-30 \% \text {, polyethylene glycol } \\
\text { dimethacrylate } 5-15 \% \text {, silane treated silica } 5 \text { - } \\
15 \% \text {, HEMA } 1-10 \% \text {, glass powder }<5 \% \text {, } \\
\text { BISGMA }<5 \% \text {, TEGDMA }<1 \% \text {. }\end{array}$ & $\begin{array}{l}\text { 3M ESPE, Dental products, } \\
\text { Seefeld-Germany }\end{array}$ \\
\hline Ketac Nano-primer & $\begin{array}{l}\text { HEMA } 35-45 \% \text {, water } 40-50 \% \text {, Vetremer } \\
\text { copolymer } 10-15 \% \text {, photoinciator. } \mathrm{pH} \approx 3\end{array}$ & $\begin{array}{l}\text { 3M ESPE, Dental products, } \\
\text { Seefeld-Germany }\end{array}$ \\
\hline Ketac Conditioner & $25 \%$ polyacrylic acid, $\mathrm{pH}=1.5-2$ & $\begin{array}{l}\text { 3M ESPE, Dental products, } \\
\text { Seefeld-Germany }\end{array}$ \\
\hline Scotchbond etchant & $35 \%$ phosphoric acid gel & $\begin{array}{l}\text { 3M ESPE, Dental products, } \\
\text { Seefeld-Germany }\end{array}$ \\
\hline $\begin{array}{l}\text { Ethylenediaminetetraacitic acid } \\
\text { (EDTA) }\end{array}$ & $\begin{array}{c}\text { Disodium EDTA } 18.87 \% \text {, Potassium Hydroxide } \\
2.62 \% \text {, Deionized water } 78.53 \% . \mathrm{pH}=7.4\end{array}$ & $\begin{array}{c}\text { Dentsply, Petropolis, } \\
\text { R.J, Brazil }\end{array}$ \\
\hline
\end{tabular}

HEMA= 2-hydroxyethyl metacrylate, BISGMA= Bisphenol A Diglycidyl Ether Dimethacrylate, TEGDMA= Triethylene Glycol Dimethacrylate.

Table 2. Means \pm standard deviations, minimum and maximum in MPa for the effect of different conditioners on SBS of nano-filled RMGI to dentin.

\begin{tabular}{lcccc}
\hline Conditioners & Means \pm SD & Min & Max & dt \\
\hline Group 1 & $5.5 \pm 2.2$ & 3.8 & 9.6 & 0 \\
Group 2 & $0.0 \pm 0.0$ & 0 & 0 & $\mathrm{c}$ \\
Group 3 & $0.0 \pm 0.0$ & 0 & 13.6 & $\mathrm{~d}$ \\
Group 4 & $8.6 \pm 3.1$ & 4.9 & 14.1 & $\mathrm{~b}$ \\
Group 5 & $12.0 \pm 1.8$ & 8.6 & 19.4 & $\mathrm{a}$ \\
\hline Group 6 & $11.9 \pm 4.4$ & 6.5 & $\mathrm{a}$ \\
\hline
\end{tabular}

$\mathrm{SD}=$ Standard deviation. $\mathrm{dt}=$ Duncan's Multiple Range Test for the effect of treatment. Min= Minimum. Max= Maximum. Means with the same letter within each column are not significantly different at $\mathrm{P}=0.05$. 
a nano-filled RMGI (Ketac Nano-100, technical material profile). This new material requires the application of nano-primer without the need for a pre-conditioning step. This reduces the number clinical application steps and consequently reduces clinical procedure time.

The use of both mild and aggressive conditioners in this study was aimed to evaluate whether the pre-conditioning step can improve the bond strength and facilitate the hybridization of the nano-primer to dentin. Based on our results, the null hypothesis was rejected. The pre-conditioning step not only significantly improved the SBS of nano-filled RMGI, but also allowed for hybridization to dentin.

In this study, occlusal dentin was used to evaluate the nano-filled RMGI/dentin interface. It was reported that when the dentinal tubules were cut obliquely, RMGI adhesive failed to form resin

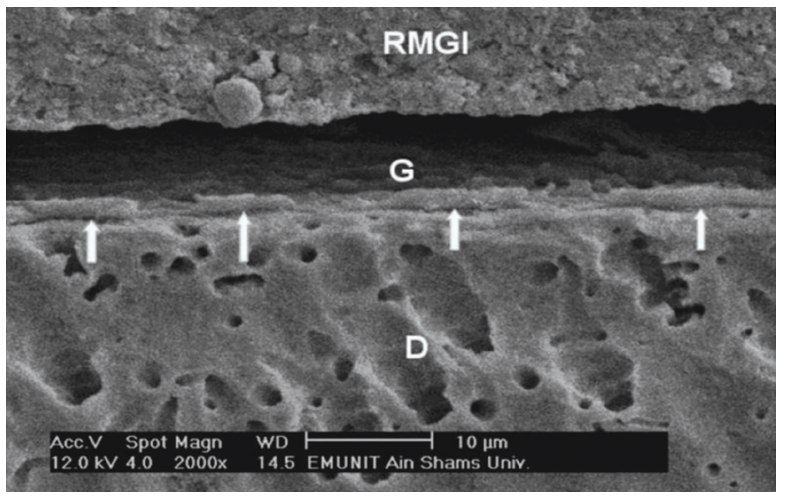

Figure 1. SEMicrograph of nano-filled RMGI/dentin interface for Group 1. No evidence of hybrid layer or resin tag extensions, with a Gap (G) between the restoration and underlying dentin. Smear layer remnants (arrows) are noticed over the dentin surface. $\mathrm{RMGI}=$ Nano-filled resin-modified glass-ionomer and $\mathrm{D}=$ Dentin.

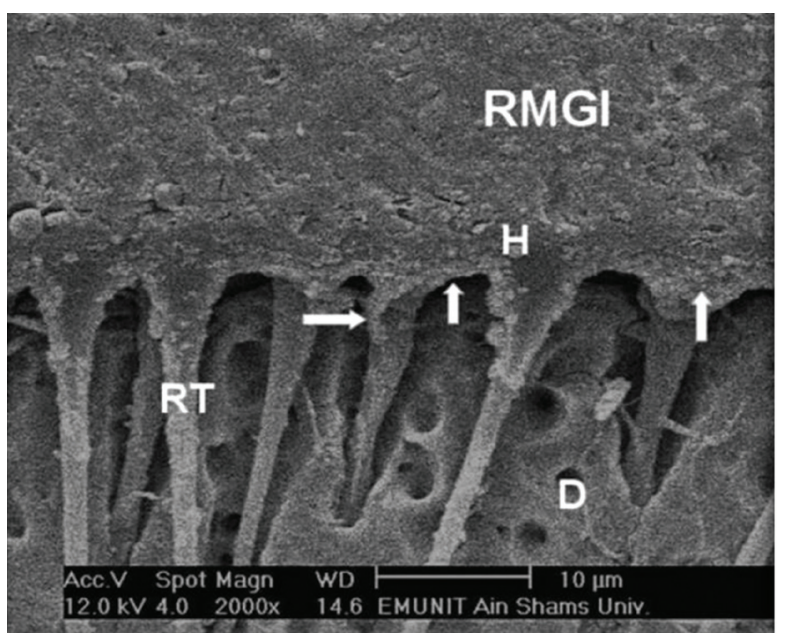

Figure 3. SEMicrograph of nano-filled RMGI/dentin interface for Group 5. Numerous long, funnel-shaped resin tag extensions (RT) with a thick hybrid layer (H). Fillers distributed at the bottom of and within the hybrid layer, as well as around the orifices of the dentinal tubules (arrows). RMGI= Nano-filled resin-modified glassionomer and $\mathrm{D}=$ Dentin. tags. ${ }^{13}$ In the occlusal dentin, the dentinal tubules were cut with an "open end," which might facilitate the penetration of the resin in the acidic primer to form resin tag extensions.

Although, the shear test, does not actually assess the adhesive bond, ${ }^{16}$ it is thus far the most popular technique to measure bond strength. The shear test is the easiest and fastest method and does not require further specimen preparations after the completion of bonding procedures. ${ }^{17}$

SEM in this study showed the presence of a smear layer over the dentin surface and the lack of hybridization formation (Figure 1), when the nano-filled RMGI was applied according to manufacturer instructions. This could be attributed to the inability of nano-primer to decalcify the underlying dentin, which might be due to the high $\mathrm{pH}$ of the nano-primer $(\approx 3)$. Our result confirmed the finding reported by Coutinho et $\mathrm{al}^{3}{ }^{3}$ despite the differ-

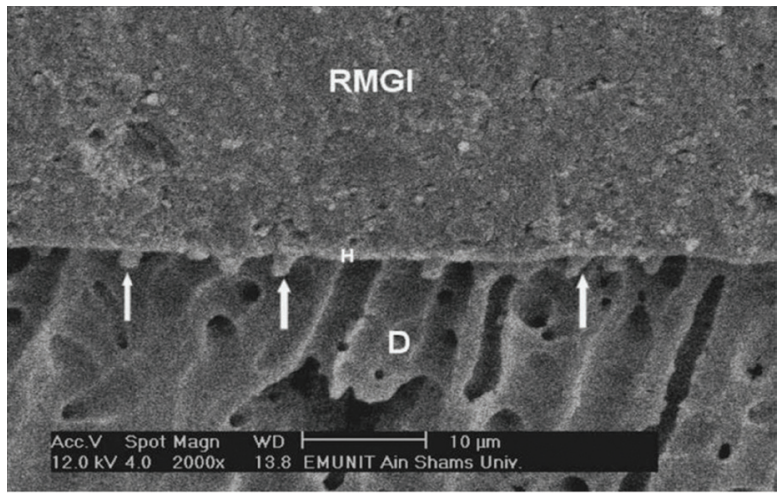

Figure 2. SEMicrograph of nano-filled RMGI/dentin interface for Group 4. Few discrete and very shallow resin tags extensions (arrows) with a thin hybrid layer $(\mathrm{H})$. RMGI= Nano-filled resin-modified glass-ionomer and $D=$ Dentin

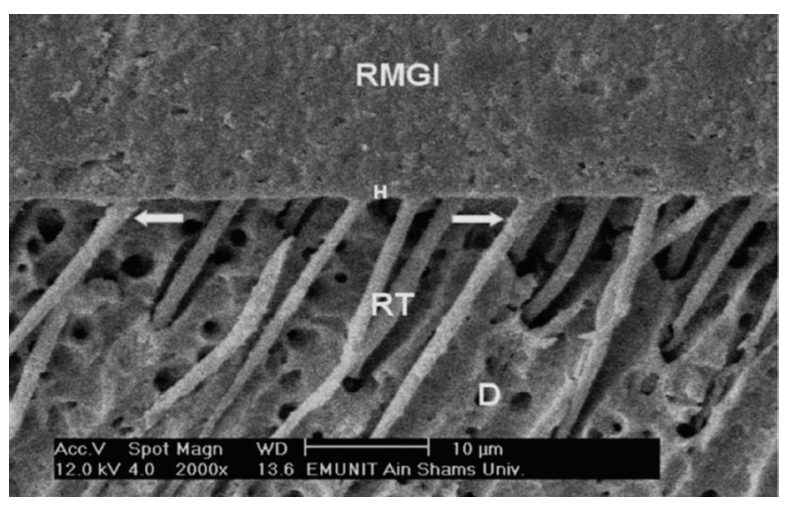

Figure 4. SEMicrograph of nano-filled RMGI/dentin interface for Group 6. Long and thin resin tag extensions (RT), the hybrid layer $(H)$ is thinner than that detected in Group 5 with a few discrete filler distributions (arrows). RMGI= Nano-filled resinmodified glass-ionomer and $\mathrm{D}=$ Dentin. 
ences in evaluation methodology between the two studies. They reported that nano-primer could not decalcify the underlying dentin surface, resulting in no evidence of hybridization. The gap noticed in SEMicrographs (Figure 1) could be attributed to the lack of hybridization and resin tag extensions in the underlying dentin, where contraction of dentin could be expected during specimen processing for SEM evaluation, which in turn allows for the separation of such material at its weaker point.

In this study, the significant improvement in the SBS of nano-filled RMGI with the pre-conditioning step could be explained by the SEM results (Figures 2, 3 and 4). It was reported that polyacrylic acid removed the smear layer without widely opening the dentinal tubules. ${ }^{18}$ Discrete and short resin tags, with the formation of the thin hybrid layer (Figure 2), might cause the significant improvement in SBS when 25\% polyacrylic acid was used.

Regardless of the difference in the aggressiveness of phosphoric acid ${ }^{19-21}$ and EDTA solution, ${ }^{11,22}$ both reported comparable SBS results. SEM showed that (Figures 3 and 4), both phosphoric acid and EDTA (Groups 5 and 6, respectively) decalcified the underlying dentin, facilitating the diffusivity of the nano-prime through the decalcified dentin surface. Phosphoric acid widely opened the dentinal tubules, resulting in the formation of numerous long and funnel-shaped, resin tag extensions (Figure 3). Furthermore, a thick hybrid layer with filler distributions at the bottom of and within the hybrid layer as well as, around the orifices of the dentinal tubules was evident (Figure 3). On the other hand, EDTA did not aggressively decalcify the dentin surface or widely open the dentinal tubules, resulting in the formation of long and thin resin tags, thinner hybrid layer, and much less filler distributions (Figure 4). It was reported that the formation of the hybrid layer and resin tag extensions is crucial to resist the acute de-bonding stresses $^{23}$ that could occur during bond strength testing. ${ }^{24}$ As different hybrid layer thicknesses were evident in SEM, given three different dentin conditioners used in this study, our SEM results suggest that the length of the resin tags played a major role in the enhancement of the SBS of nanofilled RMGI for Groups 5 and 6, when compared to all tested groups.

In the experimental design of this study, two further groups were included. One of these two groups, the nano-filled RMGI was bonded directly to the dentin surface with neither nano-primer nor dentin conditioners (Group 2) and the other was the nano-filled RMGI bonded directly on conditioned dentin surface with $25 \%$ polyacrylic acid for $10 \mathrm{sec}$ onds without the use of the nano-primer (Group 3). These two groups were included to assess whether nano-filled RMGI has the self-adhesiveness property, which is one of the main advantages of the conventional RMGI. Unfortunately, based on the SBS results of this study, nano-filled RMGI lacked the self-adhesiveness property. This result, specifically for Group 2 in this study, was consistent with the results of Coutinho et $\mathrm{al}_{1}{ }^{3}$ in spite of these studies' differences in bond strength testing methodology. They reported that when nano-filled RMGI was directly bonded to dentin, its bond was limited to the roughness that resulted from dentin surface preparation. Therefore, Groups 2 and 3 were omitted from the SEM section of the study.

Advances in material-manufacturing technology might improve some properties, but on the other hand, it could ruin other important and unique properties of the materials. Based on this fact, additional efforts should be made to carefully assess these newly introduced materials.

\section{CONCLUSIONS}

Based on the results of the current study, several conclusions can be drawn:

- The pre-conditioning step effectively improves bonding of the nano-filled RMGI to dentin.

- The self-adhesiveness of nano-filled RMGI was not proved in this study, even with the use of $25 \%$ polyacrylic acid conditioner.

- The enhancement in bonding of the nanofilled RMGI depended on the presence of long resin tag extensions in the underlying dentin.

Nevertheless, further studies should be conducted to assess the durability of nano-filled RMGI bond when a pre-conditioning step is used.

\section{ACKNOWLEDGMENTS}

The authors would like to thank Dr. E. Eid, Middle East Dental Supplies Company, Cairo, Egypt, for providing the material used in this study. 


\section{REFERENCES}

1. Inoue S, Van Meerbeek B, Abe Y, Yoshida Y, Lambrechts $P$, Vanherle G, Sano H. Effect of remaining dentin thickness and the use of conditioner on microtensile bond strength of a glass-ionomer adhesive. Dent Mater 2001;17:445-455.

2. Nicholson J, Czarnecka B. The biocompatibility of resinmodified glass-ionomer cements for dentistry. Dent Mater 2008;24:1702-1708

3. Coutinho E, Cardoso MV, De Munck J, Neves AA, Van Landuyt KL, Poitevin A Kuboki T, Yoshida Y, Suzuki K, Lambrechts $\mathrm{P}$, Van Meerbeek B. Bonding effectiveness and interfacial characterization of a nano-filled resin-modified glass-ionomer. Dent Mater 2009;25:1347-1357.

4. Peumans M, Kanumilli P, De Munck J, Van Landuyt K, Lambrechts P, Van Meerbeek B. Clinical effectiveness of contemporary adhesives: A systematic review of current clinical trials. Dent Mater 2005;21:864-881.

5. Saito S, Tosaki S, Hirota K, Characteristics of glass-ionomer cement. In: Davidson CL and Mjör IA. Advances in glass ionomer cements. Berlin: Quintessence 1999; p.1550.

6. Mazzaoui S, Burrow M, Tyas M. Fluoride release from glass ionomer cements and resin composites coated with a dentin adhesive. Dent Mater 2000;16:166-170.

7. Abdalla Al. Morphological interface between hybrid ionomers and dentin with and without smear-layer removal. $J$ Oral Rehabil 2000;9:808-814.

8. Lin A, Mclntyre N, Davidson R. Studies on the adhesion of glass ionomer cements to dentin. J Dent Res 1992;71:18361841.

9. Yoshida Y, Van Meerbeek B, Nakayama Y, Yoshioka Y, Snauwaert J, Ade Y, Lambrechts P, Vanherle G, Okazaki M. Adhesion to and decalcification of hydroxyapatite by carboxylic acids. J Dent Res 2001;80:1565-1569.

10. Jacques P, Hebling J. Effect of dentin conditioners on the microtensile bond strength of a conventional and a selfetching primer adhesive system. Dent Mater 2005;21:103109.

11. Miyasaka K, Nakabayashi N. Combination of EDTA conditioner and Phenyl-P/HEMA self-etching primer for bonding to dentin. Dent Mater 1999;15:135-157.

12. El-Askary S, Nassif M, Fawzy A. Shear bond strength of glass ionomer adhesive to dentin. Effect of smear layer thickness and different dentin conditioners. J Adhes Dent 2008;10:471-479.

13. Nakajo K, Imazato S, Takahashi Y, Kiba W, Ebisu S, Takahashi N. Fluoride released from glass-ionomer cement is responsible to inhibit the acid production of caries-related oral streptococci. Dent Mater 2009;25:703-708.
14. Toledano M, Osorio E, Osorio R, García-Godoy F. Microleakage of Class $V$ resin-modified glass ionomer and compomer restorations. J Prosth Dent 1999;81:610-618.

15. Xie D, Wu W, Puckett A, Farmer B, Mays J. Novel resin modified glass-ionomer cements with improved flexural strength and ease of handling. Europ Polymer $J$ 2004;40:343-351.

16. Della Bona A, van Noort R. Shear vs. tensile bond strength of resin composite bonded to ceramic. J Dent Res 1995;74:1591-1596.

17. Van Meerbeek B, Peumans M, Poitevin A, Mine A, Van Ende A, Neves A, De Munck J. Relationship between bond-strength tests and clinical outcomes. Dent Mater 2010;26:e100-e121.

18. Watson T. Bonding of glass-ionomer cements to tooth structure. In: Davidson $\mathrm{Cl}$ and Mjör IA. Advances in glass ionomer cements. Berlin: Quintessence 1999; p.121-136.

19. De Munck J, Van Meerbeek B, Yoshida Y, Inoue S, Susuki K, Lambrechts P. Four years water degradation of resinmodified glass-ionomer adhesive bonded to dentin. Eur $J$ Oral Sci 2004;112:73-83.

20. Kenshima S, Francci C, Reis A, Loguercio A, Filho L. Conditioning effect of dentin, resin tags and hybrid layer of different acidity self-etching adhesives applied to thick and thin smear layer. J Dent 2006;34:775-783.

21. Kenshima S, Reis A, Uceda-Gomaz N, Tancredo L, Filho L, Nogueira F, Loguercio A. Effect of smear layer thickness and $\mathrm{pH}$ of self-etching adhesive systems on the bond strength and gap formation to dentin. $J$ Adhes Dent 2005;7:117-126.

22. Fagundes T, Toledano M, Navarro M, Osorio R. Resistance to degradation of resin-modified glass-ionomer cements dentine bonds. J Dent 2009;37:342-347.

23. De Munck J, Van Landuyt K, Peumans M, Poitevin A, Lambrechts $P$, Braem M, Van Meerbeek B. A critical review of the durability of adhesion to tooth tissue: Methods and results. J Dent Res 2005;84:118-132.

24. Saber S, El-Askary F. The outcome of immediate or delayed application of a single-step self-etch adhesive to coronal dentin following the application of different endodontic irrigants. Eur J Dent 2009;3:83-89. 